\title{
Quantum Coherence and Two-Dimensional Black Holes
}

John Ellis and N.E. Mavromatos

Theory Division, CERN, CH-1211, Geneva 23, Switzerland. and

\section{D.V. Nanopoulos}

Center for Theoretical Physics, Dept. of Physics, Texas A \& M University, College Station, TX 77843-4242, USA and

Astroparticle Physics Group

Houston Advanced Research Center (HARC), The Woodlands, TX 77381, USA

and

Theory Division, CERN, CH-1211, Geneva 23, Switzerland.

\begin{abstract}
We argue that quantum coherence is maintained by two-dimensional target-space black holes in string theory thanks to an infinite set of conserved quantum numbers. These are associated with discrete solitonic string states of high spin, and may be interpreted as infinite-dimensional discrete gauge 'hair', analogous to that provided by massive axion-like fields in four dimensional theories.
\end{abstract}

ACT-41

CERN-TH.6147/91

CTP-TAMU-41/91

June 1991 
One of the key problems in theoretical physics is to reconcile quantum mechanics with general relativity. This problem may be considered as arising at two levels. The comparatively simple level of the problem is that of doing reliable perturbative calculations in a second quantised theory in a manner consistent with general coordinate invariance. This aspect of the problem seems to be solved by string theory, which incorporates general relativity and provides finite answers in perturbation theory. The more profound level of the problem is that of treating non perturbative quantum fluctuations in the topology of space-time. This aspect of the problem may also be resolved in string theory, but here our understanding is more incomplete.

There have been serious doubts raised whether conventional quantum mechanics and quantum field theory even remain valid in the presence of quantum fluctuations in the topology of space-time. Here the basic concern is that information is in general lost across event horizons, which may appear microscopically as well as macroscopically. In the macroscopic case there is a well-developed thermodynamics of black holes, according to which they have very large entropy and emit radiation thermally [1]. Macroscopic black holes forget about non-gauged quantum numbers, only have a finite set of hair in any conventional local renormalisable field theory, and achieve their large entropy by being in a mixed state. If this situation persists for microscopic fluctuations in space-time topology, one could expect pure states to evolve into mixed states [1]. This could occur only if the conventional S-matrix description of scattering broke down, and the conventional Hamiltonian evolution of the density matrix were modified. Heuristic proposals going in this direction have been made [2], which bear a passing resemblance to phenomenological ideas about the transition between quantum mechanics for small systems and classical mechanics for large systems and the quantum-mechanical collapse of the wave-function [3].

It may well be that the apparent conflict between the large entropy of the black holes and the finite number of gauge degrees of freedom in any local renormalisable field theory can be avoided if local point-like fields are replaced by extended objects such as strings. Indeed, strings offer many more types of hair, including discrete gauge symmetries [4], dualities [5], and axionic hair [6]. However, it is not yet clear whether any of these are sufficiently numerous to accommodate the large entropy associated with macroscopic black holes. Moreover, there has so far been no explicit discussion of the maintenance (or otherwise) of quantum coherence in string scattering in the presence of stringy black holes.

There are at least three string laboratories now available for addressing this question of quantum coherence. One is provided by the matrix models [7] that contain complete non-perturbative solutions, in a certain limit, of two-dimensional quantum gravity (with matter) coupled to a Liouville mode. Another is provided by the twodimensional target space black holes that can be described as $\frac{S U(1,1)}{U(1)}$ Wess-Zumino models [8]. The third is provided by time-dependent string models [9] that may be interpreted as throats of wormholes [10]. The matrix models and the two-dimensional 
string black holes are closely related, and in both cases the conventional S-matrix description appears to be adequate. In this paper we provide possible answers to the following questions: is quantum coherence indeed maintained by two-dimensional string black holes? if so, why? is it because of discrete gauge symmetries, dualities or generalised axionic hair?

Our approach starts from the observation [8] that the last stage of the black hole evaporation is described by the $\mathrm{c}=1$ matrix model, which is known to be a completely integrable theory with an infinite set of conserved quantities associated with currents of high spin [11]. In the presence of singular configurations of spacetime metrics, symmetries do not imply the existence of conserved quantities [2, 12] unless the symmetries are gauged by coupling the corresponding currents to long range fields [13]. In this paper we argue that this is just what happens in the case of two-dimensional string black holes and that, as a result, quantum coherence is maintained. We illustrate our arguments by exhibiting a conserved current that couples to a massive topological 'Q-graviton' state, and showing that this is the last phase of black hole evaporation. This is reminiscent of axionic hair, whereas duality seems to play no role.

It is instructive to review first the relevant parts of the formalism developed for the $c=1$ matrix models. The infinite set of conservation laws has been first discovered by Gross and Klebanov [11] in the fermionic representation of the model. In this language the infinite set of conserved quantum numbers of the fermions is a natural consequence of the fact that the fermions are free fields. The conserved quantities have been determined for arbitrary matrix model potentials. For illustrative purposes we restrict ourselves to the harmonic case, although our considerations are qualitatively insensitive to this. For harmonic potentials the corresponding charges read

$$
I_{n}=\int_{-\infty}^{\infty} d x \psi^{\dagger}\left(\partial_{x}^{2}+x^{2}\right)^{n} \psi
$$

The fermionic theory can be bosonised [14] and factorised in a left-right chiral form $[15,16]$. The charges may be represented in the bosonic language as

$$
I_{n}=\int_{-\infty}^{\infty} d x \int_{p_{+}}^{p_{-}} \frac{d p}{2 \pi}\left(p^{2}-x^{2}\right)^{n}
$$

where $p_{+}\left(p_{-}\right)$is the upper (lower) fermi momentum satisfying Euler's equation

$$
\partial_{t} p=x-p \partial_{x} p
$$

The fermi levels $p$ can be used to define normalised tachyonic fields of a twodimensional target space $\sigma$-model with action

$$
S=\frac{1}{4 \pi} \int d^{2} \sigma\left[\sqrt{g} g^{a b} \partial_{a} X^{M} \partial_{b} X^{N} \eta_{M N}+Q R^{(2)} \frac{q}{\sqrt{2}}+T(t, q)\right]+S_{g h o s t}
$$


where $X^{M}=(t, q)$ is a two-dimensional Minkowskian coordinate with $\mathrm{t}$ timelike and q spacelike, and $Q$ is the 'anomaly', related to the subcritical number of space-time dimensions [17], $Q=\sqrt{\frac{25-c}{3}}=2 \sqrt{2}$ (for $\left.\mathrm{c}=1\right)^{1}$. The coordinate $\mathrm{q}$ is related to $\mathrm{x}$ via

$$
x=-e^{-2 q}
$$

while the tachyonic field $\mathrm{T}$ is defined in terms of the $p$ 's as

$$
\mp \frac{p_{ \pm}}{x}=1+c\left(\partial_{q} \mp \partial_{t}-2\right) T
$$

from which the conserved quantities can be expressed in terms of the field T. In Polchinski's work [15] the quantity $c$ has been assumed to be a constant, though this may not be true in the full non-perturbative regime of the theory.

In two target space dimensions massive string states (gravitons, dilatons and in general higher rank states) are non-propagating, and one is tempted to conclude that the tachyon field is the only fully-fledged field of the theory. Indeed, the matrix model representation of the theory presented so far seems to be entirely described by this degree of freedom. However, this is not correct. There are remnants of the higher string states in two dimensions which show up already in the computation of tachyonic scattering amplitudes as an infinite set of poles with definite energy and momentum. These special states have been seen in both the continuum [18] and matrix model approaches [19], and have been interpreted by Polyakov [20] as being associated with the higher spin quasi-topological modes of the two dimensional string. They play a key role in our subsequent arguments.

To account for these higher-spin string levels one should probably go beyond the Das-Jevicki field-theoretic model [14], and consider a full closed string field theory in a non-trivial background. If such a formalism is adopted it is conceivable that the form of the conserved currents may be modified and additional stringy gauge symmetries could in principle arise. For example, it is not clear whether the constant $\mathrm{c}$ in (6) remains a constant or becomes a complicated (even non-local) function of derivatives $\partial_{q}$, as seems necessary to account for the extra poles of the tachyonic scattering amplitudes. In such a case, some of the conserved currents may be expressed as non-local functions of the various $\sigma$-model fields in target space-time.

At present it is not known how in the continuous formalism to get in a closed form the fully non-perturbative two-dimensional string effective action including the summation over genera. For illustrative purposes we therefore assume that a consistent truncation of the $\sigma$-model at genus zero and up to a certain spin level can be made. It is evident that in this way one loses symmetries of the full quantum effective theory. In the present case some of them may be attributed to the twodimensionality of space-time (e.g. it is known [21] that the charges described above

\footnotetext{
${ }^{1}$ Throughout this work we shall use units where $\alpha^{\prime}=2$.
} 
form a Cartan subalgebra of a $W_{\infty}$ algebra [22]). However, there are other gauge symmetries that survive the truncation, which are generic, in some sense, of the stringy character of the model, and usually persist even for critical strings living in higher dimensional space-times.

Let us give therefore a glance at the spectrum of the $c=1$ matter theory coupled to two-dimensional gravity. The lagrangian of the theory is described by a usual $\sigma$-model action with background fields $g^{i}$ that are generically coupled to vertex operators $V_{i}$. The latter are polynomials of arbitrary degree in $\partial_{z} X(z, \bar{z})$ and $\overline{\partial_{z}} X(z, \bar{z})$. Conformal invariance requires the $V_{i}$ to be $(1,1)$ operators, which determines the on-shell conditions. The theory necessarily has a non-trivial dilaton field, which in Liouville theory (4) is a linear function of the Liouville mode (space-like coordinate for $c \leq 1)$. The existence of a non-trivial dilaton is necessitated by the fact that the dimension of target space-time is two, while the string theory is critical, in the sense that the total central charge is 26 to compensate the ghost contribution ${ }^{2}$.

The lowest lying energy level is called a 'tachyon', although in two target space dimensions it is actually massless. In a weak field expansion around flat target spacetime, the requirement that the tachyonic emission vertex $T(k)=\exp (-\beta(k) q+i k t)$ be an operator with conformal dimension $(1,1)$ leads to the on-shell condition [17]

$$
p^{M}\left(p_{M}+Q_{M}\right)=-2
$$

where $p_{M}=(-i \beta(k), k)$ and $Q_{M}=(i Q, 0)^{3}$. The Q-term arises because of the anomaly in the expression for the world-sheet stress tensor whose operator product with the emission vertex determines the conformal properties of the latter. One can define a 'two-momentum' (for all levels) as

$$
k_{M}=\frac{Q_{M}}{2}+p_{M}
$$

The on-shell condition (7) translates into

$$
k_{M} k^{M}=0
$$

implying the masslessness of the level-0 string states.

\footnotetext{
${ }^{2}$ Alternative descriptions of the model as non-critical string theory in one dimension exist [15], but for our purposes we shall concentrate on the critical string point of view.

${ }^{3}$ Notice that the Liouville 'energies' are imaginary for central charge $c<25$. This has important consequences such as the non-existence of a one-to-one mapping between states and operators [23] which implies subtleties concerning the kind of states that propagate in intermediate channels [24]and the possibility of Liouville energy non-conservation in the corresponding amplitudes [20]. In addition, two-dimensional quantum gravity probably is not described entirely by free field methods due to boundaries in Liouville-mode space [20,25]. For our purposes we need not take into account these effects, though they may prove essential in other issues.
} 
The level-1 string multiplet consists of the graviton, a dilaton and an antisymmetric tensor. However, the usual gauge symmetry associated with the antisymmetric tensor field implies that only the field strength $H_{M N P}=\partial_{[M} B_{N P]}$ enters the effective action. In two space dimensions $H=0$, so this field can be gauged away. This is also consistent with Witten's approach to the $\sigma$ model through a Wess-Zumino gauge action [8]. The graviton vertex, describing perturbations around flat space-time, can be represented (in Fourier space) as usual by $\partial X^{M} \bar{\partial} X^{N} \exp \left(i q_{M} X^{M}\right)$. Requiring this to be a $(1,1)$ field implies the following conditions,

$$
q_{M}\left(q^{M}+Q^{M}\right)=k_{M} k^{M}-2=0
$$

and

$$
\left(q^{M}+Q^{M}\right) h_{M N}(q)=\left(k^{M}+\frac{Q^{M}}{2}\right) h_{M N}=0
$$

where $h_{M N}$ is the graviton-dilaton polarisation tensor. The constraint (11) when combined with the two-dimensionality implies the absence of transverse degrees of freedom for the graviton provided its two-momentum is not equal to $-\frac{Q_{M}}{2}$. However, for this discrete value there appears to be a 'jump' in the degrees of freedom of the system [20], in that (11) is satisfied for any $h_{M N}$. We argue below that such topological modes of high spin are important for black hole physics. It should be understood that the two-dimensional string theory has an infinity of such modes with definite energy and momenta corresponding to higher-rank string states. This can be seen readily by extending the above analysis appropriately for each mass-level.

It is worth noting that (11) is the on-shell restriction of certain recursion relations for the corresponding string amplitudes. In ordinary string theories such relations can be understood as Ward identities stemming from 'hidden' stringy gauge symmetries associated with various spin levels [26]. A similar thing happens in our case. For instance, consider a tree level amplitude involving one graviton and N-1 tachyons. The corresponding Ward identity which follows from target space-time general coordinate (canonical) transformations reads [26]

$$
\begin{gathered}
\left(k^{M}+\frac{Q^{M}}{2}\right)<V_{M N}^{G}(k) \Pi_{i=1, . . N-1} V\left(k_{i}\right)>= \\
\sum_{j=1, \ldots N-1}\left(k_{j N}-\frac{Q_{N}}{2}\right)<V\left(k+k_{j}\right) \Pi_{i \neq j} V\left(k_{i}\right)>
\end{gathered}
$$

In ordinary string theory the above identity holds even 'off-shell'. To recover $c=1$ Liouville theory we have to restrict ourselves to on-shell states. In that case the right-hand side of (12) vanishes. This is the usual 'cancelled propagator argument' in string theory. The only formal difference is the existence of the anomaly term $Q$, which from an effective field theory point of view indicates the non-triviality of the dilaton in two dimensions seen below. 
More complicated Ward indentities exist for higher spin levels, which actually lead to level mixing, as discussed by Kubota and Veneziano [27]. All these symmetries are stringy gauge symmetries associated with conserved quantities if there are no anomalies (as could be expected in certain string theories, especially in the case of two-dimensional target space-times). Following the collective first-quantised notation of Ichinose and Sakita [28], one can represent the collective string field describing arbitrary backgrounds by $\Psi$ and the corresponding world-sheet vertex operator by $V(z, \bar{z}, \Psi)$. The various stringy off-shell symmetries correspond to shifting the field $\Psi$ by a BRST-like gauge variation $Q_{B R S T} \Lambda$ and demanding symmetry of the effective action (amplitudes). In the language of conformal field theory this amounts to introducing a $Q_{B R S T}$-exact state in a given amplitude. This yields Ward identites of the form [27]

$$
\begin{aligned}
& <\hat{V}\left(z_{1}, \overline{z_{1}}, Q \Lambda\right) \Pi_{i=1, \ldots} \hat{V}\left(z_{i}, \overline{z_{i}}, \Psi\right)>= \\
& \partial_{z_{1}}<\bar{b}_{-1} V\left(z_{1}, \overline{z_{1}}, \Lambda\right) \Pi_{i=1, \ldots} \hat{V}\left(z_{i}, \overline{z_{i}}, \Psi\right)>- \\
& -\left(\partial_{z_{1}} \rightarrow \partial_{\overline{z_{1}}}, \overline{b_{1}} \rightarrow b_{-1}\right)
\end{aligned}
$$

where $\hat{V}$ is the modified vertex according to the ghost insertion rule of $[29,27]$. Upon choosing appropriate gauge functions $\Lambda$ one can recover for level one the identity (12). In this language target space-time covariance appears as one of the stringy gauge symmetries associated with the spin-two level, which in the present two-dimensional conformal theory is massive due to the anomaly Q. As already mentioned, at higher string levels one can get a mixing of levels in the corresponding Ward identities [27]. For instance, rank four tensor amplitudes are mixed with rank three and two (graviton-dilaton) amplitudes. Consequently, the form of the conservation laws becomes more involved.

Now we come to our main point. In the presence of singular space-time backgrounds such as black holes, such conservation laws do not in general lead to conserved quantities (generalised charges), and this results in the problems mentioned in the introduction concerning the loss of quantum coherence and the nonfactorisability of the scattering matrix when topological fluctuations of the spacetime metric are encountered. In Euclidean target-space formulations of the present theory, one can imagine the formation of baby-universe-like configurations which could contribute to the loss of quantum coherence in the presence of fluctuations around a black hole classical solution. We shall now argue that the enormous stringy symmetries (which are enhanced in our case due to the two-dimensionality) suppress any such loss of information, since the conserved currents are coupled to the stringy quasi-topological discrete modes and remain conserved even during the Hawking proccess of black hole evaporation. Unfortunately, we cannot formally prove our arguments due to the non-existence, as yet, of a satisfactory string field theory in curved background space-times. However, we shall illustrate our mechanism by restricting our attention to the first massive level, at genus zero. As we have seen, the gauge symmetry associated with this level incarnates target space general coor- 
dinate invariance, which implies the conservation of a spin-two current, the stress tensor.

We shall restrict ourselves to one $\sigma$-model loop order, and comment briefly on the extension to higher orders, which is facilitated enormously with the help of field redefinitions, due to the particular dimensionality of space-time. In terms of the coset conformal field theory considered by Witten [8], working to first order in $\alpha^{\prime}$ amounts to taking the large $k$ limit, where $k$ is the coefficient of the Wess-Zumino term in the ancestor gauge conformal field theory. It is in this limit that the black hole solution has been analysed [8]. It might be possible to find exact solutions which could still maintain the basic features of the black hole, but the situation is not clear ${ }^{4}$. The effective field theory truncated to the first massive level and to order $\alpha^{\prime}$ (second order in derivatives) reads [31]

$$
\int d^{2} x \sqrt{G} e^{\phi}\left(R+G^{i j} \nabla_{i} \phi \nabla_{j} \phi+G^{i j} \nabla_{i} T \nabla_{j} T+\Lambda+V(T)\right)+\ldots
$$

where $\Lambda$ is a (target space) cosmological constant originating from the non-critical dimensionality of space-time. A few comments are in order concerning the nontrivial scale factor in front of the Einstein term and its special role in two space-time dimensions. Usually in higher dimensional field theories such factors can be absorbed by suitable conformal transformations of the metric. In this way one recovers the usual Einstein term for the scalar curvature. However, in two dimensions the form (14) is more fundamental. One can never transform away the scale factor once it is non-trivial. Indeed, under a generic conformal transformation in D space-time dimensions of the form $G_{i j}^{\prime}=e^{c \phi} G_{i j}$ the curvature tensor transforms as,

$$
R^{\prime}=e^{-c \phi}\left(R-c(D-1) \nabla^{2} \phi-\frac{1}{4}(D-1)(D-2) G^{i j} \partial_{i} \phi \partial_{j} \phi\right)
$$

For $\mathrm{D}=2$ the combination of the Einstein term with the scale factor is conformally invariant. This is the main reason for the appearance of the extra Q-terms in the otherwise familiar conservation laws (11) etc. (for linear dilaton terms this is the result of a differentiation of the scale factor). We also note that by choosing $c=-1$ one can transform away the dilaton kinetic terms in (14). This is the scheme we shall adopt from now on, unless otherwise stated.

Now let us focus the attention on the stress tensor of the theory. As usual we define it as a response of the 'matter' action to a variation of the gravitational field. We are mainly interested in the effects of the black hole solution on the conservation of the charge associated with this spin-two current. The graviton equation of motion reads

\footnotetext{
${ }^{4}$ The exact solution claimed in [30] correponds to a thermal bath in Euclidean formalism, which does not represent the physically interesting case associated with collapsing matter that we consider in this work.
} 


$$
\left(R_{M N}-\frac{1}{2} G_{M N} R\right) e^{\phi}=T_{M N}^{(T)}+T_{M N}^{(\phi)}
$$

where the index $(T)((\phi))$ on the stress tensor labels the corresponding parts referring to the tachyon (dilaton). The dilaton stress-tensor includes the cosmological constant piece, and it can easily be computed as

$$
\begin{aligned}
T_{M N}^{(\phi)}-\frac{1}{2} G_{M N} \Lambda & =e^{\phi}\left[G_{M N}\left(\nabla^{2} \phi+\left(\nabla_{K} \phi\right)^{2}\right)-\nabla_{M} \phi \nabla_{N} \phi-\nabla_{M} \nabla_{N} \phi\right] \\
& =G_{M N} \nabla^{2}\left(e^{\phi}\right)-\nabla_{M} \nabla_{N}\left(e^{\phi}\right)
\end{aligned}
$$

In two dimensions the left hand side of (16) vanishes identically. This implies that the combination, $T_{M N}$, of the tachyonic stress tensor and the cosmological constant part of the dilaton energy-momentum tensor is itself a total space-time divergence for the black hole solution $[8]^{5}$. This in turn implies the following conservation law

$$
\nabla^{M} T_{M N}=\frac{1}{2} \nabla_{N} \phi R e^{\phi}
$$

Asymptotically the space-time is flat for the black hole solution [8], so the tachyonic spin-two current is conserved (the cosmological constant part of $T$ is trivially conserved). One can, therefore, define a conserved charge (asymptotically),

$$
\int_{\text {space }} T_{o o}=-\int_{\text {space }} G_{o o}^{\text {black-hole }} \nabla_{\rho}^{2}\left(e^{\phi^{\text {black-hole }}}\right)
$$

where we took into account the fact that the black hole solution is a static solution of space-time with coordinates $(t, \rho)$.

The above analysis can easily be extended to higher orders in $\alpha^{\prime}$ by exploiting the freedom one has in performing local redefinitions of fields. The latter correspond to different choices of renormalisation schemes for the evaluation of the $\sigma$-model $\beta$ functions [32]. As long as one truncates the theory to a certain (finite) order in the $\sigma$-model loop expansion the locality of the effective action and the corresponding field redefinitions is guaranteed. For instance, from general tensor analysis of the string effective action in $D=2$ it becomes clear [33] that there is always a scheme in which the order $\left(\alpha^{\prime}\right)^{2}$ stringy corrections to the effective action contain, in addition to the terms in (14), only graviton-tachyon mixed terms of the form

$$
\int \sqrt{G} e^{\phi} R^{2}(T+\text { const })
$$

\footnotetext{
${ }^{5}$ We assume, following [8], that the tachyonic field does not destroy the black hole solution of the pure graviton-dilaton sector of the theory, but only produces marginal deformations, and a classical 'hair' determined by the value of the Liouville (world-sheet) cosmological constant. The latter is itself produced by the tachyon boundary values in Liouville space $[25,20]$.
} 
(remember that in $D=2$ the only inequivalent structure of the curvature tensor is the Ricci scalar). By performing a constant shift in the tachyon field we can always consider such terms as part of the 'matter' action, which leaves the dilaton stress tensor as before ${ }^{6}$.

The above considerations show clearly that the spin-two current leads to a conserved charge (asymptotically) that is a surface term in space (in two dimensions there is a degeneracy since the boundary of one-dimensional space consists only of points). Hence its conservation will be maintained by the Hawking process of black hole evaporation. According to our previous considerations, the stress tensor is the current associated with a gauge symmetry of the string characterising the level-one massive string multiplet. The corresponding charge will help characterise the black hole.

This situation is reminiscent of axionic hair in four-dimensional black holes. There the axion charge is a surface term defined as $\int_{V(\Sigma)} H=\int_{\Sigma} B$, where $V(\Sigma)$ is a three space bounded by $\Sigma$, and $B_{M N}$ is the antisymmetric tensor field, whose dual is a scalar in four dimensions. The axion can become massive through topological couplings with $U(1)$ gauge field one-forms $A$ : $\int B \wedge d A$, and the gauge fields themselves become massive by eating the only degree of freedom of the B field. In the case we are considering, the role of the long-range fields is played by the discrete graviton state with momentum $-\frac{Q^{M}}{2}$. This is the only remnant of the gravitational multiplet in two space-time dimensions, since any propagating graviton would be longitudinal and, as such, gauged away through the Ward identity (11). The pertinent coupling in the effective action is provided by the classical trace of the stress tensor $T_{M N}$ constructed from the lagrangian (14). The tachyonic and cosmological constant parts of the effective lagrangian then can be rewritten in the form,

$$
\int d^{2} x \sqrt{G}\left[e^{\phi}(\nabla T)^{2}+G^{M N} T_{M N}\right]
$$

On-shell, the stress-tensor trace term is a total divergence exactly as happens with the corresponding topological mass term for the axion-gauge coupling. Such surface terms become non-trivial for space-times with non-trivial topologies, such as the Schwarzschild metric spaces. Of course there are differences from the axion case in the way the charges are measured. In axion-like couplings a charge cannot be measured by point-like particles, since the latter are coupled only to the axion field strength which vanishes outside the black hole. Hence Bohm-Aharonov type experiments with strings (cosmic or fundamental) are needed [6]. This is not the case with the stress-tensor example, but it might well be that some of the other stringy gauge charges exhibit such non-classical behaviour.

\footnotetext{
${ }^{6}$ This could also be understood by performing redefinitions of the dilaton field appearing in the scale factor of the metric, which is always present in $D=2$. The latter method does not produce any shifts in the cosmological constant and is more suitable when the tachyonic coupling to $R^{2}$ has the form of a generic scalar function $f(T)$.
} 
The physics of the Hawking process is now clear. The final stage of black hole evaporation excites the quasi-topological Q-graviton and higher-spin discrete states, which couple to the exact quantum numbers of the asymptotic theory. This coupling produces an exact conservation of this quantum numbers during the Hawking process and one recovers this set in the matrix model as remnants of the black hole.

It is straightforward to show formally that the final stage of the black hole excites Q-graviton modes. This can be seen most easily in the so-called Schwarzschild gauge studied in [34] ${ }^{7}$. This gauge implies a dilaton linear in the space-like coordinate (Liouville mode), $\phi=Q \rho$. The black hole assumes the familiar Schwarzschild form,

$$
d s^{2}=-g(\rho) d t^{2}+\frac{1}{g(\rho)} d \rho^{2} ; g(\rho)=1-a e^{Q \rho}
$$

The parameter $a$ is related to the mass of the black hole. At the latest stage of the Hawking process the mass $a$ becomes very small. In this limit $d s^{2}=-d t^{2}+d \rho^{2}+$ $a e^{Q \rho}\left(d t^{2}+d \rho^{2}\right)$, and the metric perturbation becomes identical to that of a vertex operator exciting a $\mathrm{Q}$-graviton state. This state cannot be gauged away in the matrix model and constitutes what Polyakov [20] calls a 'jump in the degrees of freedom at exceptional values of momenta'. In the presence of tachyonic backgrounds there may be excited additional remnants of the graviton state, those which arise as poles in the tachyonic amplitudes for $c=1$ models $[18]^{8}$. All these should be considered as traces of the black hole that have survived the evaporation process. In the full theory one expects the excitation of all the discrete higher spin states, which are the only remnants in two dimensions of the entire string spectrum.

For the particular case of two dimensions one expects to find - in addition to symmetries that one would encounter in any ordinary string theory - extra symmetries associated with the specific dimensionality of space-time (e.g. extended conformal algebras etc.). It seems that this is the case with the infinitely many conserved charges of the fermion theory [21]. If these symmetries are local in form then the corresponding currents must couple to topological graviton (and in general highspin) modes, as is the case of the tachyon stress tensor. The massive character of the extra states (of the order of the string Planck mass) does not affect the arguments in favour of conservation, due to the quasi-topological nature of these fields. One can even draw a rough analogy between these states and soliton solutions in ordinary string theories with non-trivial dilaton backgrounds [9]. These models are also related to Wess-Zumino conformal theories [35], and may be interpreted as describing topologically non trivial configurations in space-time, like half of wormhole

\footnotetext{
${ }^{7}$ To make direct contact with the relevant literature we work now in the original formalism without performing a conformal field redefinition of the space-time metric.

${ }^{8}$ In the flat target space-time formalism the poles appear to correspond to on-shell tachyons, in the sense of (7). But this might be deceptive, given the non-triviality of the black hole space-time. It appears that the only correct interpretation for these extra poles is to think of them as remnants of high-spin string states in the presence of non-trivial tachyon backgrounds in two dimensions.
} 
throats [10]. Thus, the infinite set of exactly conserved discrete charges that characterises the $c=1$ matrix model (viewed as a two-dimensional critical string theory) probably characterises the black hole.

The values of the conserved charges are in principle independent of the black hole mass, and a fixed-mass black hole can have arbitrary charges of this kind. This is the only point where the stress tensor example is not a generic one, since this particular spin-two current is related to the dilaton stress tensor and therefore to the total energy of the black hole. However, due to the cosmological constant piece, the charge corresponding to this current is non-vanishing even in the limiting (zeromass) black hole case. Since there is no propagating graviton degree of freedom in two dimensions, the existence of the conserved charge that we find is a non-trivial manifestation of a stringy gauge symmetry. In this way the simplest arguments in favour of the loss of quantum coherence are evaded, given the enormous symmetries carried by the stringy black holes. By this we mean that the set of conserved charges is complete in characterising the quantum-mechanical states of the two dimensional problem.

Some of these charges, such as, for instance, the fermion number corresponding to $n=0$ in (1), might not be easy to identify in the $\sigma$-model theory. Despite that, it becomes clear from its definition $(2,6)$ that the fermion number charge can be expressed, as expected from general arguments due to the locality of the corresponding current, as a surface term in space (determined by the Liouville mode $\rho=q \sqrt{2}), \int_{\text {space }} d \rho \sqrt{\hat{G}} \nabla_{\rho}\left[e^{-\frac{Q}{2} \rho}(T+\right.$ const $\left.)\right]$. The exponent is clearly related to the asymptotic form of the dilaton. The same dilaton scale factors enter the expression for the space-time metric $\hat{G}[9]$. The symmetry associated with this number in the $\sigma$ model is not yet fully understood. However, we believe that all of these symmetries, manifest in the fermionic representation of the asymptotic theory, are related to stringy BRST-like symmetries for various multiplets, as is the case for the spin-two current examined in detail above. It is conceivable, for example, that the possibility of always finding a renormalisation scheme where the form of the tachyon potential in the effective theory is quadratic in the tachyon field [36], reflects such a stringy symmetry for the lowest-lying string level (tachyon), which in $D=2$ is associated with the fermion number. Of course, as far as this particular charge is concerned, formal coupling to long-range fields in the effective action might not be necessary for its exact conservation in the presence of singular metric backgrounds. This point needs further clarification.

An alternative scenario, again in favour of the maintenance of quantum coherence in the two-dimensional black hole case, is the possibility that some of the currents are non-local in space-time. This is a totally unfamiliar picture, but it cannot be excluded, as we have mentioned earlier. Such a non-locality could probably ensure the exact conservation of the corresponding charge during the Hawking process, 
in a way similar to what a coupling of the conserved current to a long range-field does for conventional local theories. It is understood that in such a case a formal coupling with the topological modes might not be necessary. However, even this exotic possibility can be attributed to the stringy effects of the black hole. The matrix model is, after all, believed to be a stringy regularisation scheme of continuous Liouville theory in which the summation over genera is done exactly.

A final point we would like to comment upon concerns the duality symmetry that characterises general string theories [37]. In the case of two-dimensional black holes, such duality symmetries can be interpreted $[38,39,30]$ as arising from the gauging of different subgroups of $S U(1,1)$ (vector or axial). In Minkowskian formalism, their effect is to interchange the horizon of the black hole with the singularity in the Kruskal diagram. However, the dual black hole has itself its own singularities, so loss of information could in principle occur, and the duality symmetry in question seems incapable of solving the problem of quantum incoherence. Other obstacles to the possibility of getting black hole quantum hair from duality symmetries in ordinary string theories have been noted in [40]. Of course, at present our understanding of duality symmetry may not be sufficient to reveal its importance for the problem, and it may turn out that generalised duality symmetries [5, 41] are indeed essential for space-time physics.

We have argued that stringy symmetries are essential in providing enough hair to restore quantum coherence and thereby reconcile quantum mechanics and black hole physics. We have seen that gauge symmetries in $(1+1)$-dimensional string theory lead to exactly conserved quantities that are respected even by the Hawking process. The key to this was the coupling of the corresponding currents to discrete remnants of higher-spin states. This scenario leads us to imagine analogous scenaria that might operate in higher space-time-dimensional theories. The infinite set of symmetries coupled to discrete modes in the two-dimensional case might translate, for higherdimensional theories, into the existence of infinitely large discrete quantum hair for stringy black holes provided by the breaking of certain gauge symmetries down to discrete subgroups (gauged discrete symmetries) [4]. As argued recently [42], it is only gauged discrete symmetries (as is the case of strings) that can have non-trivial dynamical effects on black hole physics. This particular type of quantum hair might manifest itself through axionic-like couplings between currents and long-range gauge fields (that exist naturally in string theories). It may well be, following the example set by the two-dimensional case, that the entire massive string spectrum provides quantum hair for black holes, which is sufficient to maintain coherence during the evaporation process. By 'sufficient' we mean, again, that the quantum mechanical states can be classified completely by those charges.

These points are of course mere speculations at this stage. To establish them one might have to wait for the development of a consistent string field theory. This is a point where toy string laboratories, like the matrix models, might prove useful. 
They might provide one with prototypes of the non-perturbative stringy calculations needed to answer such obscure questions as the reconciliation of quantum mechanics and gravity, not to mention the origin of space-time.

\section{Acknowledgements}

We thank L. Alvarez-Gaumé, I. Antoniadis, C. Bachas, S. Kalara, G. Lazarides, D. Lüst and G. Veneziano for discussions. The work of D.V.N. is partially supported by DOE grant DE-FG05-91-ER-40633. 


\section{References}

[1] S. Hawking, Comm. of Math. Phys. 43 (1975), 199.

[2] J. Ellis, J.S. Hagelin, D.V. Nanopoulos and M. Srednicki, Nucl. Phys. B241 (1984), 381.

[3] G. Ghirardi, D. Rimini and T. Weber, Phys. Rev. D34 (1986), 470.

[4] L.M. Krauss and F. Wilczek, Phys. Rev. Lett. 62 (1989), 124;

J. Preskill and L. M. Krauss, Nucl. Phys. B341 (1990), 50.

[5] S. Kalara and D. V. Nanopoulos, Texas preprint CTP-TAMU-14/91-ACT-28 (1991).

[6] M.J. Bowick, S.B. Giddings, J.A. Harvey and A. Strominger, Phys. Rev. Lett. 61 (1988), 2823.

[7] D.J. Gross and A.A. Migdal, Phys. Rev. Lett. 64 (1990), 717;

M. Douglas and S. Shenker, Nucl. Phys. B335 (1990), 635;

E. Brezin and V. Kazakov, Phys. Lett. 236B (1990), 144.

[8] E. Witten, Institute for Advanced Study, Princeton preprint IASSNS-HEP$91 / 12$.

[9] I. Antoniadis, C. Bachas, J. Ellis and D.V. Nanopoulos, Nucl. Phys. B328 (1989), 1546.

[10] C. Callan, J. Harvey and A. Strominger, Enrico Fermi Institute preprint EFI91-03 (1991).

[11] D.J. Gross and I.R. Klebanov, Nucl. Phys. B352 (1991), 671.

[12] D.J. Gross, Nucl. Phys. B236 (1984), 349.

[13] T.J. Allen, M.J. Bowick and A. Lahiri, Phys Lett. 237B (1990), 47.

[14] S.R. Das and A. Jevicki, Mod. Phys. Lett. A5 (1990), 1639.

[15] J. Polchinski, University of Texas preprint UTTG-06-91 (1991).

[16] D.J. Gross and I.R. Klebanov, Princeton University preprint PUPT-1241 (1991).

[17] F. David, Mod. Phys. Lett. A3 (1988), 1651;

J. Distler and H. Kawai, Nucl. Phys. B321 (1989), 509. 
[18] D.J. Gross, I.R. Klebanov and M.J. Newman, Nucl. Phys. B350 (1991), 621 ; P. Di Francesco and D. Kutasov, Princeton University preprint PUPT-1237 (1991).

[19] U. H. Danielsson and D.J. Gross, Princeton University preprint PUPT-1258 (1991).

[20] A.M. Polyakov, Mod. Phys. Lett. A6 (1991), 635.

[21] J. Avan and A. Jevicki, Brown University preprint BROWN-HET-801 (1991).

[22] C.N. Pope, L.J. Romans and X. Shen, Phys. Lett. B236 (1990), 173; Nucl Phys. B339 (1990), 191.

I. Bakas and E. Kiritsis, LBL, Berkeley preprint LBL-29394 (1990).

[23] N. Seiberg, Rutgers University preprint RU-90-29 (1990), to appear in the Proc. of the 1990 Yukawa International Seminar, Common Trends in Mathematics and Quantum Field Theories.

[24] G. Moore, Yale-Rutgers Universities preprint YCTP-P8-91,RU-91-12 (1991).

[25] J. Polchinski, Nucl. Phys. B346 (1990), 253.

[26] G. Veneziano, Phys. Lett. 167B (1985), 388;

J. Maharana and G. Veneziano, Nucl. Phys. B283 (1987), 126.

[27] G. Veneziano, unpublished ; T. Kubota, Stringy Symmetries and Off-Shell Ward Identities, talk on B. Sakita's 60th birthday (1989).

[28] I. Ichinose and B. Sakita, Phys. Lett. 175B (1986), 423.

[29] J. Polchinski, Nucl. Phys. B307 (1988), 61.

[30] R. Dijkgraaf, E. Verlinde and H. Verlinde, Princeton preprint PUPT-1252, IASSNS-HEP-91/22 (1991).

[31] C. Callan, D. Friedan, E. Martinec and M. Perry, Nucl. Phys. B262 (1985), 593; E.S. Fradkin and A.A. Tseytlin, Nucl. Phys. B261 (1985), 1.

[32] D.J. Gross and E. Witten, Nucl. Phys. B277 (1986), 1;

A.A. Tseytlin, Phys. Lett. 176B (1986), 92.

[33] A.A. Tseytlin, John Hopkins University preprint JHU-TIPAC-91004 (1991).

[34] G. Mandal, A. Sengupta and S. Wadia, Institute for Advanced Study, Princeton preprint IASSNS-10-91 (1991). 
[35] I. Antoniadis, C. Bachas, J. Ellis and D.V. Nanopoulos, Phys. Lett. 211B (1988), 393.

[36] T. Banks, Rutgers University RU-91-08 preprint (1991).

[37] K. Kikkawa and M. Yamasaki, Phys. Lett. 149B (1984), 357;

N. Sakai and I. Senda, Progr. Theor. Phys. 75 (1986), 692;

V. Nair, A. Shapere, A. Strominger and F. Wilczek, Nucl. Phys. B287 (1987), 402 ;

A. Shapere and F. Wilczek, Nucl. Phys. B320 (1989), 669.

S. Ferrara, D. Lüst, A. Shapere and S. Theisen, Phys. Lett. B225 (1989), 363.

A. Giveon, E. Rabinovici and G. Veneziano, Nucl. Phys. B322 (1989), 167.

A. Giveon and M. Porrati, Phys. Lett. 246B (1990), 54.

[38] A. Giveon, LBL, Berkeley preprint LBL-30671 (1991).

[39] E.B. Kiritsis, University of California, Berkeley preprint UCB-PTH-91-21 (1991).

[40] J. H. Schwarz, Caltech preprint CALT-68-1728 (1991).

[41] G. Veneziano, CERN preprint CERN-TH-6077/91 (1991).

[42] S. Coleman, J. Preskill and F. Wilczek, Institute for Advanced Study, Princeton preprint IASSNS-HEP-91/17 (1991). 